\title{
Effectiveness of a tier 3 weight management programme for children and young people in a deprived area
}

\author{
R. P. G. Hayhoe ${ }^{1}$, M. Haddow ${ }^{2}$, J. Shareef ${ }^{2}$, S. Barnes ${ }^{3}$ and A. A. Welch ${ }^{1}$ \\ ${ }^{1}$ Population Health and Primary Care, Norwich Medical School, University of East Anglia, Norwich, \\ Norfolk NR4 7TJ, ${ }^{2}$ Healthy Weight Team, James Paget University Hospital, Lowestoft Rd, Great Yarmouth, \\ Norfolk NR31 6LA and ${ }^{3}$ Public Health, Norfolk County Council, County Hall, Martineau Lane, \\ Norwich NR1 2DH
}

Childhood obesity is a major public health concern which has seen a rapid upward trend since the $1980 \mathrm{~s}^{(1)}$. The long-term impact on health is potentially severe as it frequently tracks into adulthood ${ }^{(2)}$. Comorbidities traditionally associated with adult obesity are now commonly seen in obese children, including cardiovascular disease risk factors, type 2 diabetes, musculoskeletal issues, and respiratory complications such as sleep apnoea and asthma ${ }^{(3)}$.

The Healthy Weight tier 3 weight management service provides a novel specialist programme for obese children and young people (CYP) in Great Yarmouth and Waveney, where obesity prevalence is significantly higher than East of England and national averages $^{(4)}$. This population group has significant deprivation, and prevalence of behavioural and learning difficulties, which together with difficult family situations are likely to be contributing to increased risk of obesity, as well as presenting barriers to engagement and effective weight management. The multi-component programme follows NICE guidelines (CG43) and is available to all local CYP with BMI $>98^{\text {th }}$ percentile for their age (UK90 reference cohort), or $>91^{\text {st }}$ percentile with complex needs. The focus is on healthy lifestyle promotion by regular weight measurement, weight management discussion, dietary advice, and physical activity referral. Provision is tailored to each individual, based on specialist clinical assessment.

Change in obesity status was assessed at follow-up time points of 3, 6, 9, 12, 18 and 24 months. Standard protocols were used to measure height and waist circumference and a Tanita BC420MA was used to measure total weight and bioelectrical impedence-estimated muscle mass. BMI z-scores were used as the primary outcome measure as recommended by NICE guidance (CG47), with waist-height ratio (WHtR) and fat free muscle index (FFMI), providing more detail of the change in body weight and composition achieved.

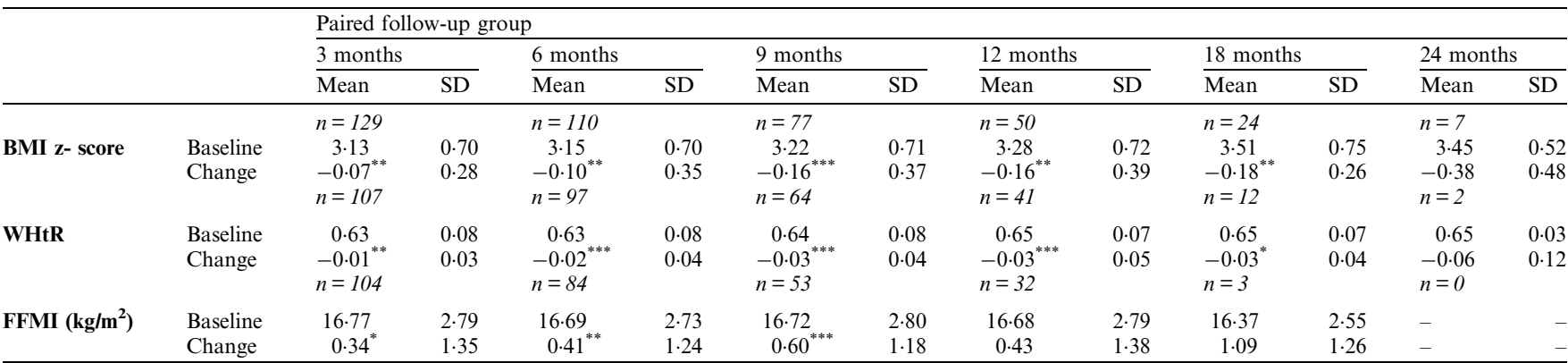

${ }^{*} p<0.05,{ }^{* *} p<0 \cdot 01,{ }^{* * *} p<0.001$ versus baseline (two-tailed paired sample t-test). Baseline WHtR and FFMI measurement may not have been at first appointment

The mean age of those enrolled in the service was 10.8 (SD 4.0) and 60\% were girls. 64\% resided in areas ranked in the two most deprived national quintiles according to the 2010 Index of Multiple Deprivation. Statistically significant mean reductions in paired BMI z-scores and WHtR were evident at all follow-up points 3-18 months. Together with increases in FFMI this demonstrates improvement in obesity status and body composition.

Although this was an evaluation without a control group for comparison, results suggest this unique service has provided a successful programme of obesity treatment, monitoring and onward referral, to very obese CYP in Great Yarmouth and Waveney. Given the complexities of engaging this population the positive changes observed here are a significant achievement and provide support for implementation of similar programmes elsewhere.

1. POST (2003) Parliamentary Office of Science and Technology POSTnote 03/205.

2. Serdula MK, Ivery D, Coates RJ, et al. (1993) Prev Med 22, 167-177.

3. Ebbeling CB, Pawlak DB and Ludwig DS (2002) Lancet 360, 473-482.

4. Public Health England (2013) Fingertips Public Health Profiles, available at fingertips.phe.org.uk. 